\title{
Report
}

\section{Discussion and Training to Health Professionals on IMNCI 2015}

\author{
Hadgu Gerensea*, Tadis Brhane \\ School of Nursing, Aksum University, Aksum, Ethiopia \\ Email address: \\ hadgugerensea2015@gmail.com (H. Gerensea)
}

\section{To cite this article:}

Hadgu Gerensea, Tadis Brhane. Discussion and Training to Health Professionals on IMNCI 2015. Advances in Surgical Sciences. Vol. 3, No. 6, 2015, pp. 37-41. doi: 10.11648/j.ass.20150306.11

\begin{abstract}
Ethiopia, with 74 million people, has $6^{\text {th }}$ highest number of child death in the world. Every year half a million children under five die. More than $70 \%$ of these deaths occur from the five IMCI targeted diseases namely acute respiratory infections (28\%), malaria (20\%) diarrhea $(20 \%)$ and malnutrition $(55 \%)$ and often in combination. Therefore, integrated Management new born and Childhood Illness (IMNCI) strategy is an evidence-based cost effective strategy for managing common childhood illnesses. Similarly, our needs assessment finding on child health services in under-five OPD in ten sample health center selected among five affiliated practice sites of CHS-AKU shows low implementation of IMNCI due to high turnover of trained staffs, high transfer rate of the trained health providers from place to place and mismanagement of the trained staffs at the health center level i.e. these trained was not assigned in the intended site/ for intended specific task. Therefore, the Objective of this project is: To provide in-service training for 20 health professional and to conduct forum discussion with 20 stake holders in affiliated practice site of CHS-AKU on IMNCI to equip the health workers with necessary knowledge and skill management of common childhood illnesses and to strengthen the existing under five child services in all aspects. This project will improve case management in practice site of CHS-AKU by equipping with necessary knowledge and skills to deal IMNCI strategy. It has also improving the practical training settings where students are undergoing professional practice guided by exemplary trained/skilled/ health professionals in practice site -CHS- AKU.
\end{abstract}

Keywords: IMNCI, Forum, Training, CHS-AKU

\section{Introduction}

\subsection{Background Information}

Axum University is the youngest pioneer training institute for health care professionals in the country. It is a center of excellence for both in-service and pre-service training of health professionals, health care service delivery and research. The college has a number of staff that can provide high quality service and training.

\subsection{Statement of the Problem}

Over the last 3 decades the annual number of deaths among children less than 5 years of age has decreased by almost a third. However, this reduction has not been evenly distributed throughout the world. Every year more than 10 million children die in developing countries before they reach their fifth birthday. Seven in 10 of these deaths are due to acute respiratory infections (mostly pneumonia), diarrhea, measles, malaria, or malnutrition - and often to a combination of these illnesses. [1]

Worldwide infant Mortality rate continues to be high at 68/1000 live births and Under Five Mortality Rate at $95 / 1000$ live births per year. Neonatal mortality contributes to over $64 \%$ of infant deaths and most of these deaths occur during the first week of life. Mortality rate in the second month of life is also higher than at later ages [2,3].Common childhood illnesses will continue to be major contributors to child deaths through the year 2020 unless greater efforts are made to control them. This assumption makes a strong case for introducing new strategies to significantly reduce child mortality and improve child health and development.

Ethiopia, with 74 million people, has 6th highest number of child death in the world. Every year half a million children 
under five die. More than $70 \%$ of these deaths occur from the five IMCI targeted diseases namely acute respiratory infections (28\%), malaria (20\%) diarrhea $(20 \%)$ and malnutrition (55\%) and often in combination [4].

Quality of care is another important indicator of inequities in child health. Every day, millions of parents seek health care for their sick children, taking them to hospitals, health centers, pharmacists, doctors, and traditional healers. Surveys reveal that many sick children are not properly assessed and treated by these health providers, and that their parents are poorly advised. At first-level health facilities in low-income countries, diagnostic supports such as radiology and laboratory services are minimal or non-existent, and drugs and equipment are often scarce. Limited supplies and equipment, combined with an irregular flow of patients, leave health professionals at this level with few opportunities to practice complicated clinical procedures. Instead, they often rely on history and clinical signs to determine a course of management that makes the best use of available resources. Providing quality care to sick children in these conditions is a serious challenge. Yet how can this situation be improved [3].

Experience and scientific evidence show that improvements in child health are not necessarily dependent on the use of sophisticated and expensive technologies, but rather on effective strategies that are based on a holistic approach, are available to the majority of those in need, and which take into account the capacity and structure of health systems, as well as traditions and beliefs in the community.

Integrated Management of Childhood Illness (IMCI) Strategy is an evidence-based proven cost effective strategy for managing common childhood illnesses. So the objectives of this training will be to provide in-service training for health professional and to conduct forum discussion with stake holders in affiliated practice site of CHS-AKU to equip the health workers with necessary knowledge and skill how to manage common childhood illnesses and to strengthen the existing under five children services which will contribute our country strategy to reduce two-third of childhood mortality to achieve Millennium Goal development.

\subsection{Justifications}

Ethiopia health indicator (2010) revealed that infant and child mortality rate in Ethiopia were 59 and 88 per 1000 live breath respectively whereas 6 and 5 per 1000 in industrial countries. This accounts $50 \%$ of the total recorded death in Ethiopia. More than $70 \%$ of these deaths occur from the five IMCI targeted diseases namely acute respiratory infections (28\%), malaria (20\%) diarrhea (20\%) and malnutrition (55\%) and often in combination $[4,5]$.

Generally this training has the following significance

- It is cost effective in limited resources countries like Ethiopia i.e. does not need sophisticated and expensive technologies

- It supplements under-five OPD by trained heath care providers in order to provide better case management approach.

- Improving the practical training settings where students are undergoing professional practice guided by exemplary trained/skilled/ health professionals in practice site -CHSAKU.

- Helps to solve the acute shortage of IMNCI trained H.W mentioned in the need assessment due to high turnover of trained staffs.

\section{Objectives}

\subsection{General Objective}

To provide in-service training for 20 health professional and to conduct forum discussion with 20 stake holders in affiliated practice site of CHS- AKU on IMNCI to equip the health workers with necessary knowledge and skill of management of common childhood illnesses and strengthen the existing under five children services in all aspects which will contribute our country strategy to reduce two-third of childhood mortality to achieve Millennium Goal development.

\subsection{Specific Objectives}

- To familiarize health professional in practice site (health centers) of CHS- AKU with WHO IMNCI guidelines and formats to be used for managing common childhood illness.

- To equip health professional in practice site of CHS- AKU with necessary knowledge that will enable them to manage common childhood illnesses.

- To improve the skills of health workers in practice site of CHS- AKU for better case management in health facilities.

\section{Methodology}

\subsection{Project Area}

Five hospitals namely (St. marry Aksum hospital, Adwa hospital, Axum University Referral hospital, Birshwa general hospital and Suhl hospitals) and eight health centers namely (Wukiro Health center, Selekleka health center, Endagermen health center, Shire health center, Millennium health center, Aksum health center, Adwa health center and Edaga Arbi health center) directors were discussed with panel type of discussion on the challenges and solutions of implementation of IMNCI guideline on prevention and management of childhood illness.

\subsection{Project Design}

Conduct forum discussion with stake holders and providing in-service training with to health professionals

\subsection{Strategy}

Select and train 20 health Professional who have assigned in child health service in affiliated practice site of CHS- AKU (Axum town (millennium and Axum Health center), Wikiro district ( 2 health center), Selekileka district ( 2 health center), Shire town (Germen and shire health center) and Adwa district ( 2 health center).

Conducting meeting with 20 stakeholders' included; 
community health extension workers, head of district health office, head of each health center and local NGO to create awareness and to gain support on the implementation of the program. Establish technical working group at each district and health center level that will provide technical direction and supervision to the implementation, consistency and sustainability of the program. Create strong linkage with health extension workers, head of district health office, head of each health center and local NGO gradually to take over and maintain the all the in service program. Provide supportive supervision every a month at least for a year to ensure implementation of the program according to IMNCI strategy. Distribute two copy of IMNCI material support (IMNCI chart booklet, IMNCI registration book and other format) to each Health center/health institution.

\section{Result}

\subsection{Phase-One: Forum Discussion with Stake Holders (01/09/2015)}

Total of 15 directorate and 5 Chief Executive Officers (CEO) from hospitals were included in the forum discussion. The forum discussion was given for full one day on Exoduse Hotel. The forum discussion shows there is a problem on Implementation of IMNCI guideline. These problems are focused on shortage of health professionals, luck of skill and knowledge. Previously different NGO were supported the programme but know the NGO were phase-out.

The common reasons mentioned by the Participants for nonimplementation of IMNCI strategy were:

- High turnover of trained staffs.

- High transfer rate of the trained health providers from place to place.

- Mismanagement of the trained staffs at the health center level i.e. These trained was not assigned in the intended site

- Shortage of medical equipment

These mentioned problems are similar with need assessment finding.

As some problems are managed by the Directorate and CEO they reach mutual agreement to solve the problem from its grass root level.

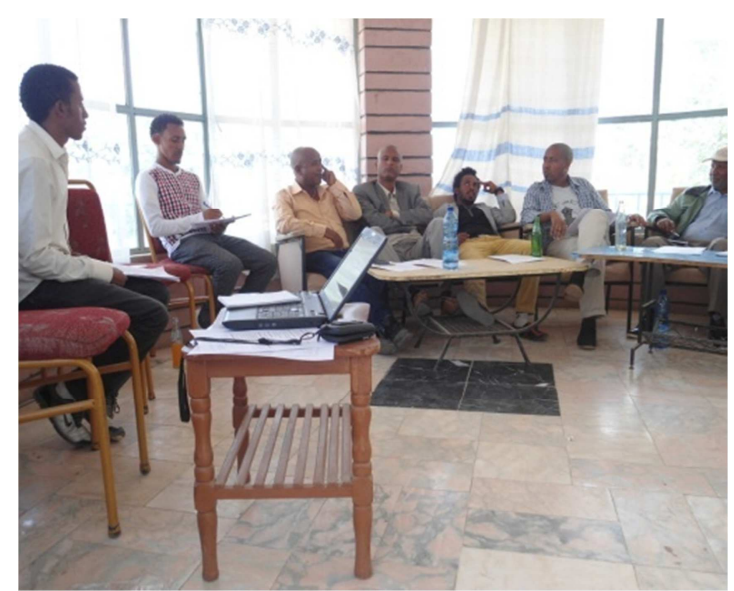

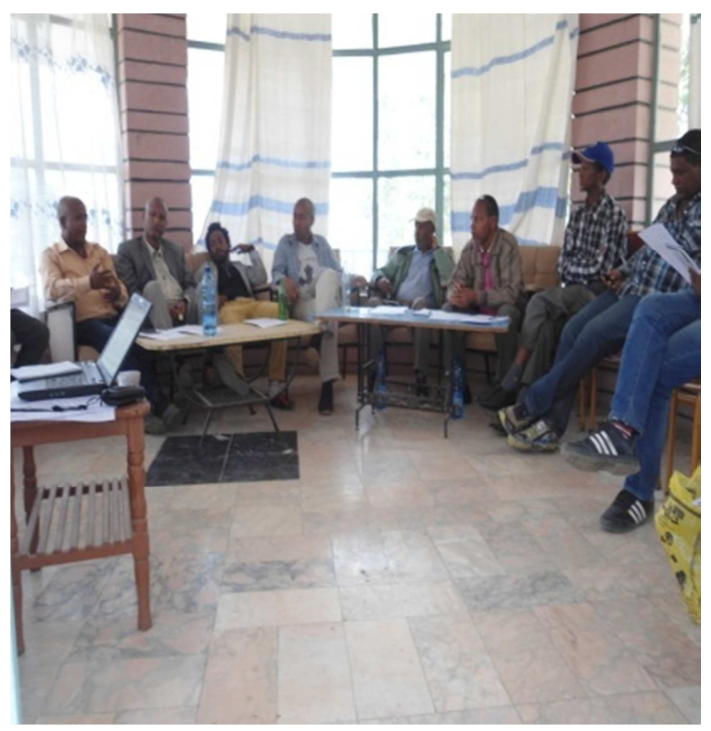



Fig. 1. Forum discussion with Chief Executive Officers and Directorates in affiliated sites of AkU-CHS in Exoduse hotel May 01-02/2015.

\subsection{Phase-Two: Conducting IMNCI Training (03-11/09/2007)}

This is the main objective of the project:

Intensive training for 20 health professional from hospitals and health centers which are from Central and North West Zone of Tigray region. The training was given for 7 days by skilled full health professionals who have TOT on IMNCI guideline of 2013.

For each trainee the new guideline was given to improve the resource on each health center and hospital. Similarly to equip the material for professionals with day to day activities in their working environment.

The training was effective as the post test result as most results were above $80 \%$.

We distribute two copy of IMNCI material support (IMNCI chart booklet) to each Health center/health institution. But registration book was not given as TRHB have no accesses of this guideline. 
Number of proper filling and documentation of IMNCI registration book in practice site (during supervision most of the registration guideline was filled appropriately.

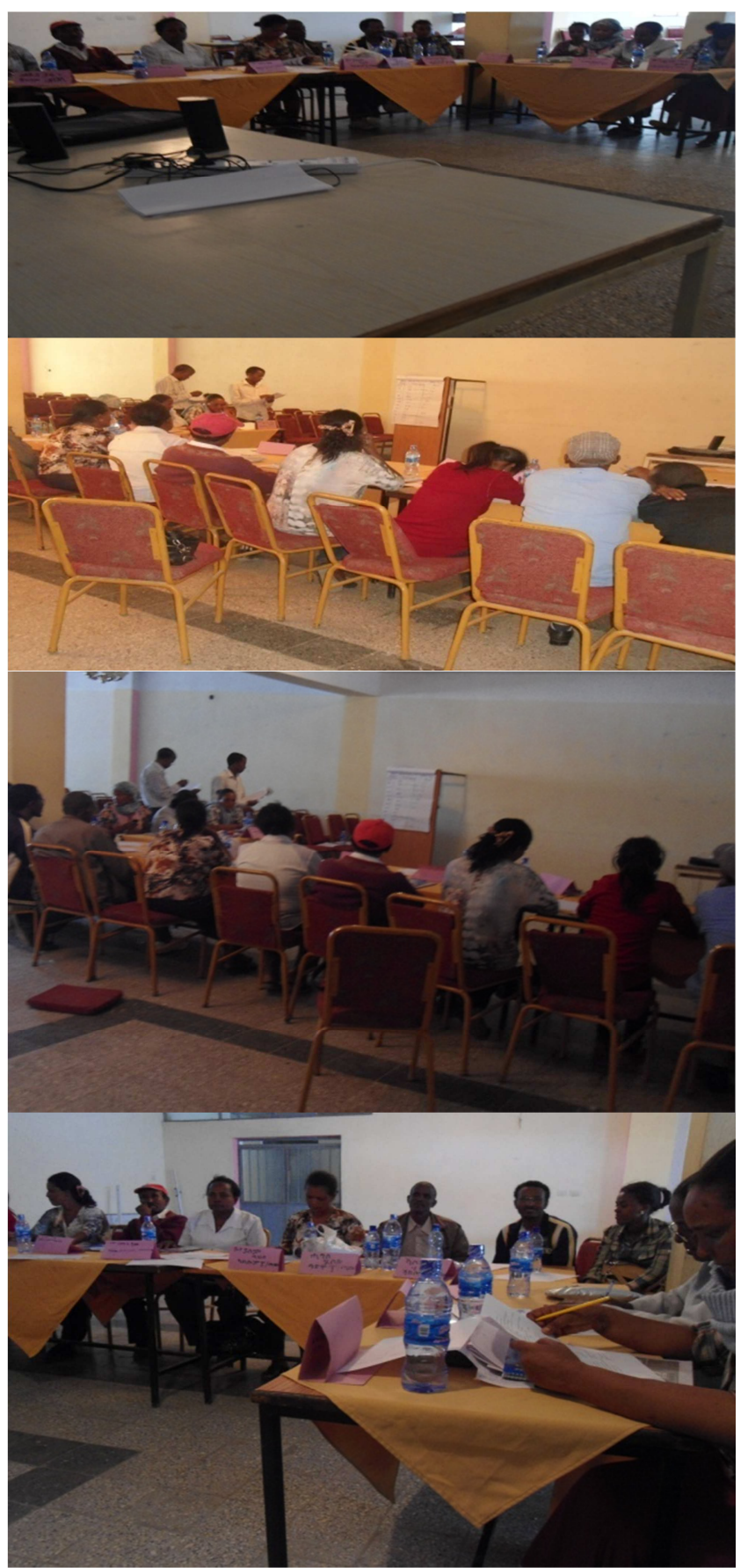

Fig. 2. Training on IMNCI for health professionals in affiliated sites of AkU-CHS in Exoduse hotel May 03-11/2015.

\subsection{Phase-Three: Monitoring and Supervision:(13-19/09/2007)}

Each health centers and hospitals in affiliated practice site of CHS-AKU were supervised their implementation of IMNCI Guideline after intensive training.

During supervision some health centers were not implemented properly and corrective measures and discussion with directorates were conducted.

But in most hospitals IMNCI guideline was not still implemented. This gap is still persistent since Hospitals staffs are Resistance to change and their attitude towards the guideline was not satisfactory. During the supervision we try to solve some problems but sill it needs extensive forum discussion with health professionals.

\subsection{Key Indicators for Achievement}

Pre and post test training score more than $70 \%$.

Number of trained health workers in practice site of CHS-AKU are 20

Number under five OPD at health center level managed by IMNCI trained H.W (all health centers and hospitals except Adwa hospital who is absent during the supervision.

Number of participating stakeholders in forum discussion are 20.

Number of distributed IMNCI material: only IMNCI chart booklet was distributed as Registration book was missed from TRHB.

Number of regular meeting with district health professional: forum discussion with stake holders was conducted (20 participants)

Project program progress evaluation as compared to base line assessment (outcome of the project) was satisfactory:

Especially all health centers are used IMNCI guideline for prevention and management of childhood illness. But hospitals are still lack this approach of management. Hospitals staff are Resistance to change. This persisting problem is still managed through continues training and forum discussion with health professionals.

\section{Conclusion}

Starting from phase one an integrated and subsequent intervention was given for central and west north zones of Tigray region hospitals and health centers on IMNCI. Five hospitals namely (St. marry Aksum hospital, Adwa hospital, Axum University Referral hospital, Birshwa general hospital and Suhl hospitals) and eight health centers namely(Wukiro Health center, Selekleka health center, Endagermen health center, Shire health center, Millennium health center, Aksum health center, Adwa health center and Edaga Arbi health center) directors were discussed with panel type of discussion on the challenges and solutions of implementation of IMNCI guideline on prevention and management of childhood illness. Similarly, Training for health professionals from each hospitals and health centers were conducted from May 03-11/2015 on The Updated guideline of IMNCI 2013. After training was given close supervision and monitoring was given for each hospital and health center for six days. Some corrective measures were taken on Axum health center and shire health center. But till all hospitals except Birshwa they did not use the guideline because of highly skilled health professionals and most cases are referral. Similarly their attitude on the guideline was unsatisfactory. So, close supervision and till forum discussion is requested for hospitals. 


\section{References}

[1] World Health Organization. World Health Report 1999 making a difference. Geneva, WHO, 1999.

[2] Murray CJL and Lopez AD. The global burden of disease: a comprehensive assessment of mortality and disability from diseases injures, and risk factors in 1990 and projected to 2020 . Geneva, World Health Organization, 1996.

[3] World Health Organization. Report of the Division of Child Health and Development 1996-1997. Geneva, WHO, 1998.

[4] Ethiopian Demographic and Health survey (EDHS), 2005.

[5] Ministry of health of Ethiopia, Health and health related indicators2006/2007. Planning andprogramming department, $\mathrm{FMoH}$, Addis Ababa, Ethiopia. 